\title{
Neurostimulation of the Cholinergic Antiinflammatory Pathway in Rheumatoid Arthritis and Inflammatory Bowel Disease
}

\author{
Yaakov A Levine, ${ }_{1}^{1}$ Frieda Koopman, ${ }^{2}$ Michael Faltys, ${ }_{1}^{1}$ Ralph Zitnik, ${ }^{1}$ and Paul-Peter Tak ${ }^{3,4,5}$ \\ ${ }^{1}$ Setpoint Medical Corporation, Valencia, California, United States of America; ${ }^{2}$ Academic Medical Centre/University of Amsterdam, \\ Amsterdam, the Netherlands; ${ }^{3}$ Ghent University, Ghent, Belgium; ${ }^{4}$ Cambridge University, Cambridge, United Kingdom; and \\ ${ }^{5}$ GlaxoSmithKline, Stevenage, United Kingdom
}

\begin{abstract}
Inflammatory bowel disease (IBD) and rheumatoid arthritis (RA) cause significant morbidity and mortality. Despite significant therapeutic advances, the medical need for patients with these disorders remains high. An important neural-immune regulatory mechanism termed the "inflammatory reflex," and its efferent arm, the "cholinergic antiinflammatory pathway" regulate innate and adaptive immunity. An emerging body of evidence indicates that stimulation of this pathway with implantable medical devices is a feasible therapeutic approach in disorders of dysregulated inflammation. Herein we describe the underlying biology and the preclinical experiments done in standard animal models that provided the rationale for testing in clinical trials. The preclinical development approach comprised elements of classic drug and medical device development, yet had unique features and challenges. "Bioelectronic medicines" having ideal characteristics of both drugs and medical devices hold great conceptual promise for treatment of systemic diseases in the future. However studies being done today will help determine whether neurostimulation of the cholinergic antiinflammatory pathway (NCAP) has the potential in the nearer term to fulfill the needs of patients, caregivers and payers for an additional potential treatment option for inflammatory disorders, and might thus become one of the first feasible examples of a bioelectronic medicine.
\end{abstract}

Online address: www.bioelecmed.org

doi: 10.15424/bioelectronmed.2014.00008

\section{INFLAMMATORY BOWEL DISEASE AND RHEUMATOID ARTHRITIS: CLINICAL CHARACTERISTICS AND CURRENT TREATMENT APPROACH}

Inflammatory bowel disease (IBD), comprising Crohn's disease and ulcerative colitis, and rheumatoid arthritis (RA) are debilitating chronic inflammatory disorders that together affect approximately $1 \%$ to $1.5 \%$ of the population in developed countries. These diseases have a major negative impact on patients' lives and an enormous social and economic cost as they often reach their greatest severity during what should be the highly productive years of early adulthood and middle age $(1,2)$. In the case of RA, it is increasingly appreciated that incompletely treated systemic inflammation leads to acceleration of atherosclerosis with elevated risk of morbidity and mortality from cardiovascular disease (3). Both IBD and RA are typically treated in a step-care paradigm, beginning with older drugs such as corticosteroids and immunosuppressive agents (for example, azathioprine in Crohn's disease and methotrexate in RA). Although many patients can be controlled

Address correspondence to Paul P Tak, GlaxoSmithKline, Gunnels Wood Road, Stevenage SG1 2NY, United Kingdom. Phone: +44-1438-763766; E-mail: paul-peter.x.tak@gsk.com. Submitted October 7, 2014; Accepted for publication October 9, 2014; Published Online (www.bioelecmed.org) December 2, 2014.

The Feinstein Institute for Medical Research Empowering Imagination. Pioneering Discovery. on these drugs, most will progress to targeted biological or small molecule therapies including first line agents such as tumor necrosis factor (TNF) inhibitors, followed by a variety of second-line agents. Although these targeted therapies are quite efficacious and have resulted in major improvements in disease control, not all patients respond; and they are associated with significant side effects and are costly, so there remains a significant unmet medical need for patients with RA and IBD.

\section{A POTENTIAL ALTERNATIVE APPROACH TO RA AND IBD TREATMENT: THE INFLAMMATORY REFLEX AND THE CHOLINERGIC ANTIINFLAMMATORY PATHWAY}

The vagus nerve mediates the inflammatory reflex, a mechanism the central nervous system utilizes to regulate innate and adaptive immunity (4). The afferent arm of the reflex senses inflamma- 
tion both peripherally and in the central nervous system, and downregulates the inflammation via efferent neural outflow. The efferent arm of this reflex has been termed the "cholinergic antiinflammatory pathway" (CAP) (Figure 1). The reflex serves as a physiological regulator of inflammation by responding to environmental injury, pathogens and other external threats with an appropriate degree of immune system activation $(4,5)$.

Our understanding of the CAP began two decades ago with the first studies showing communication between the brain and the immune system $(6,7)$. Tracey and colleagues demonstrated that systemic, hepatic, and splenic TNF production as well as the physiological manifestations of endotoxemic shock in rodents were worsened by vagotomy and ameliorated by electrical stimulation of the cervical vagus nerve (VNS). Further, based on in vitro experiments, they postulated that this effect was mediated directly by acetylcholine acting through a specific $\alpha-7$ nicotinic acetylcholine receptor $(\alpha 7 \mathrm{nAChR})$ on macrophages (8). It was later demonstrated that reducing the response to endotoxemia using NCAP required an intact spleen, and selective anatomical lesion experiments showed that an intact neural pathway to the spleen from the cervical vagus through the celiac plexus was also necessary for this effect (9). Within the spleen itself, nerve fiber synaptic vesicles are found in close apposition to TNFsecreting macrophages (10), and to a recently described population of cluster of differentiation (CD) $4^{+} \mathrm{CD} 44^{\text {high }} \mathrm{CD} 62^{\text {low }}$ $\mathrm{T}$ cells (11). These T cells bear surface $\beta$ adrenoceptors, and have the capacity to synthesize and secrete acetylcholine. This unique T-cell population functions analogously to an intermediate neuron, sensing adrenergic neurotransmitters released by the splenic nerve, and, in turn, transmitting the efferent signal to adjoining splenic macrophages by local secretion of acetylcholine. The $\alpha 7 n A C h R$ expressed on the surface of macrophages is essential for the NCAP effect, as demonstrated by antisense oligonucleotide, targeted disruption (12) and adoptive transfer experiments (13).

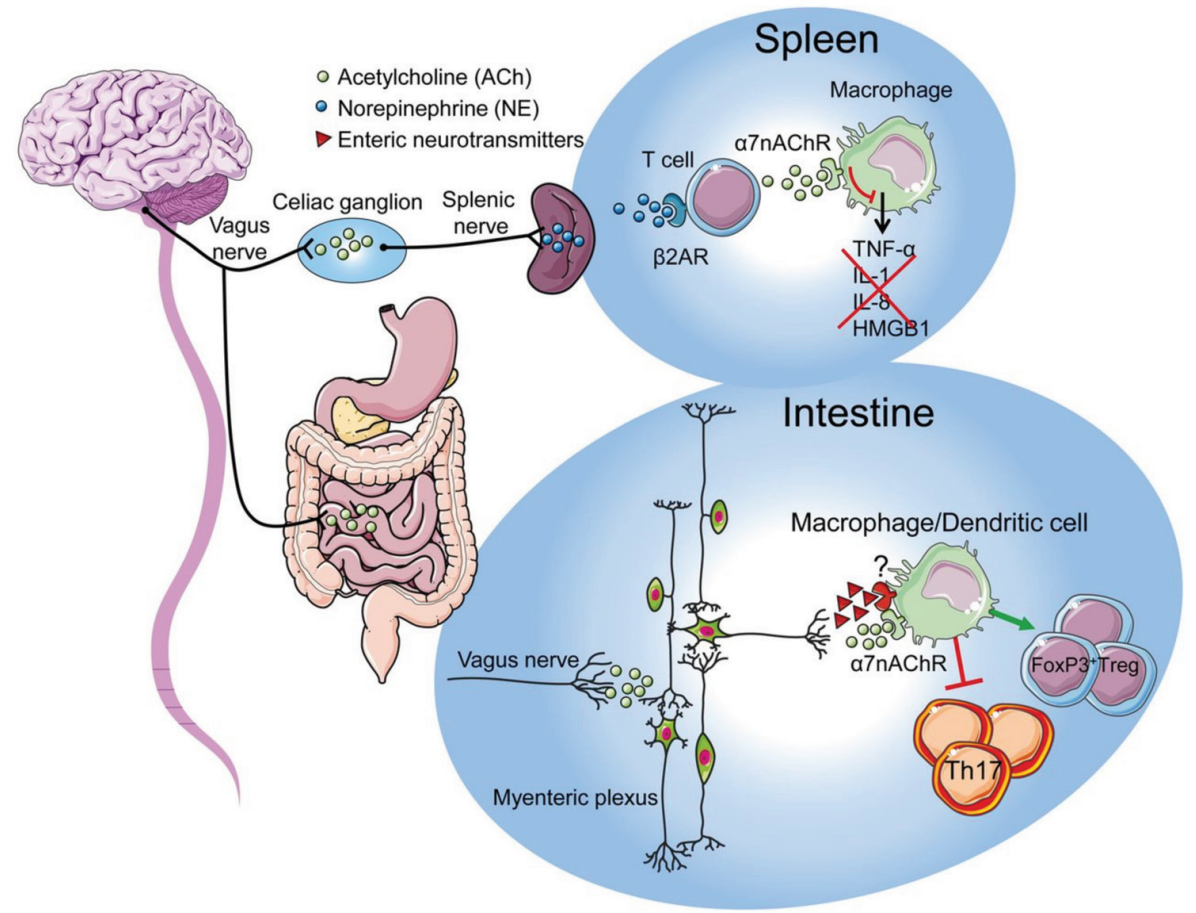

Figure 1. Schematic representation of the cholinergic antiinflammatory pathway. The reflex is activated in the circumventricular brainstem and vagal afferent fibers through signaling by inflammatory mediators. After integration, this sensory input will trigger the efferent arm via the celiac ganglion, modulating the immune response in the spleen. Adrenergic neurons in the spleen release norepinephrine that induces acetylcholine release by a subset of splenic T cells. Acetylcholine ligands the $\alpha 7 n A C h R$ expressed on cytokine-producing macrophages thereby reducing the release of TNF, IL-1, IL-18, HMGB1, and other cytokines. During intestinal inflammation the vagal efferent fibers directly contact myenteric neurons in the intestinal wall. Enteric neurons release acetylcholine, similarly reducing intestinal macrophage cytokine release. Image from (14) (reproduced from Gut, The vagal innervation of the gut and immune homeostasis, Gianluca Matteoli, Guy E Boeckxstaens, 62:1214-22, @ 2013, with permission from BMJ Publishing Group Ltd.).

In contrast to the path through the spleen, vagal efferent fibers directly innervate the gut with the exception of the distal colon, synapsing with the myenteric plexus without an intermediate anatomic ganglion (see Figure 1). Nerve fibers lie in close apposition to macrophages and dendritic cells in the gut wall. Intermediary acetylcholine-producing $\mathrm{T}$ cells analogous to those observed in the spleen have not yet been identified (14). Indeed, in a model of postoperative inflammatory ileus, VNS was able to reduce intestinal inflammation even in recombinaseactivating gene (RAG)-1 knockout animals lacking $\mathrm{T}$ cells, and in animals with surgical denervation of the spleen. Inflammation in this model was not reduced by
VNS in animals with targeted disruption of the $\alpha 7 \mathrm{nAChR}$, but this deficit could be reconstituted by bone marrow transplant from wild-type animals (15). In contrast, the spleen was required for resolution of mucosal inflammation by central cholinergic agonists in a recent study in experimental colitis (16). Vagus nerve terminals have been shown to interface with cholinergic myenteric fibers, yet the question of precisely which if any nonneuronal intermediate cells complete the pathway between the vagus and macrophages in the gut is still being resolved (15).

Circulating monocytes produce TNF and other proinflammatory cytokines in response to in vitro exposure to bacterial lipopolysaccharide (LPS) in normal ro- 
dents, normal human volunteers and RA patients. After VNS or in vitro exposure to cholinergic agonists, the ability of these peripheral blood cells to release inflammatory mediators in response to LPS is reduced markedly $(17,18)$. In rodent models, VNS reduced granulocytic infiltration to sites of injury, including the muscularis mucosa in inflammatory postoperative ileus (19), to the pancreas in pancreatitis (20), and to the lung following burninduced acute lung injury (21). Experiments using carrageenan to induce inflammation demonstrated that VNS or pharmacologic CAP reduced trafficking to sites of inflammation, driven by a reduction in surface expression of the integrin component CD11b on neutrophils (22).

When taken together, these observations demonstrate that CAP activation reduces production of systemically active cytokines, chemokines and antibodies by resident spleen cells, and causes circulating cells which traverse the spleen to develop an altered phenotype with reduced expression of inflammatory mediators and adhesion molecules. As a result of this altered phenotype, trafficking of immune cells to inflamed tissue is reduced. Further, upon entering inflamed tissue, these cells are less able to release mediators that both directly damage tissue, and induce other cells to migrate and cause damage secondarily.

An increasing body of evidence indicates that the CAP also can be harnessed to reduce pathological inflammation. Neurostimulation of the cholinergic antiinflammatory pathway (NCAP) using VNS may be a feasible means of treating diseases including RA and IBD that are characterized by excessive and dysregulated inflammation $(4,5,23)$. Implantable medical devices are increasingly being studied and approved for use as therapies for systemic diseases that, in the past, were traditionally treated with medicines. One such application would utilize a chronically implanted neurostimulator to reduce systemic and organ-specific inflammation in diseases including IBD and RA, potentially offering a treatment alternative to patients, physicians and healthcare payers (24) (Figure 2).

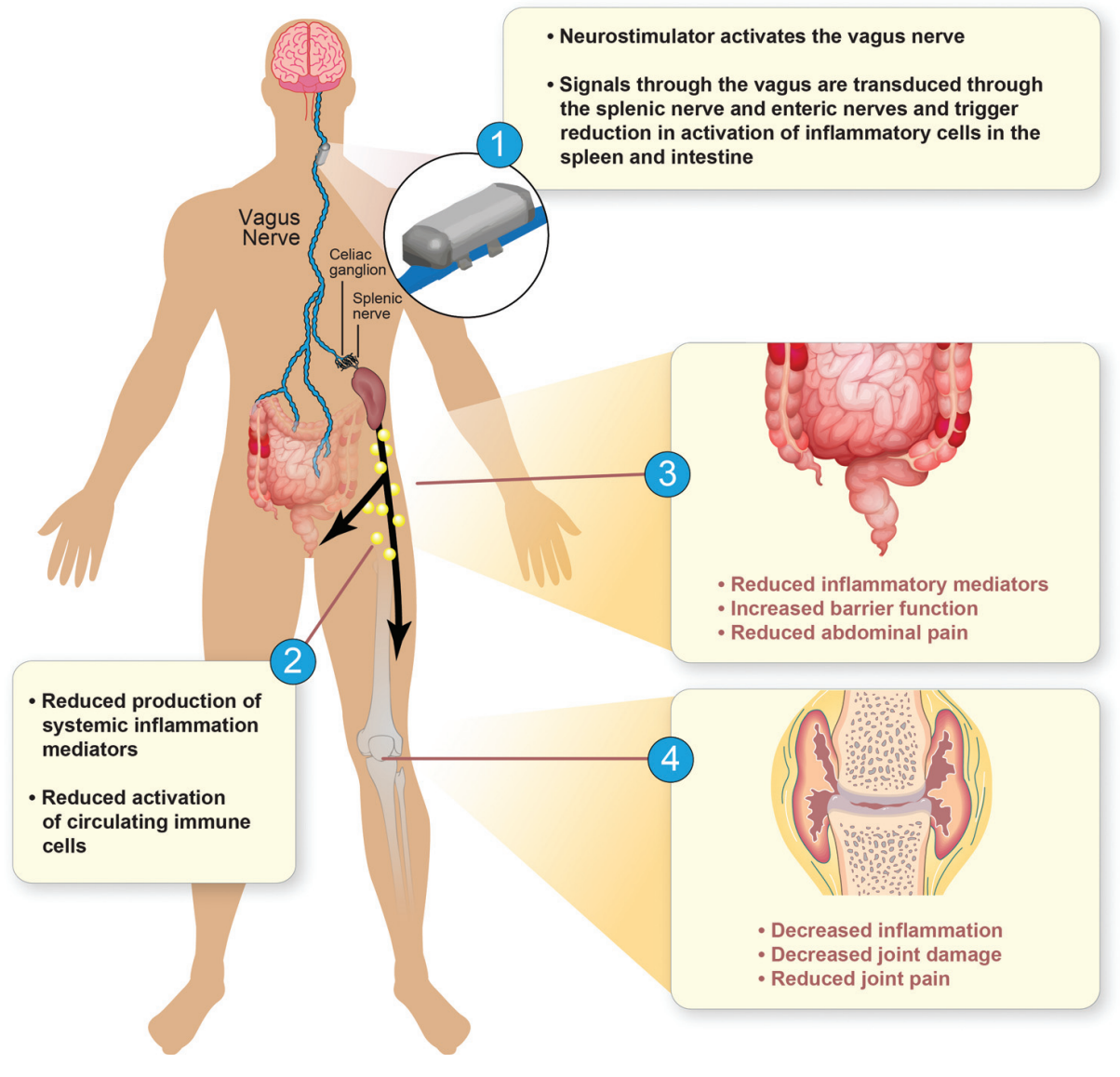

Figure 2. Neurostimulation of the cholinergic antiinflammatory pathway with an implantable medical device: A potential alterative therapeutic approach for RA and IBD. Signals from the device pass through the efferent vagus nerve and are relayed directly to the intestine, and across the celiac ganglion, traversing the splenic nerve to terminate in the spleen (panel 1). Neurotransmitter release in the end organs results in reduced activation of resident immune cells (panel 1), as well as reduced production of inflammatory mediators and activation of circulating immune cells (panel 2). These effects should result in attenuation of inflammation with improvement in signs and symptoms, and reduction in joint and intestinal mucosal damage (panels 3 and 4).

\section{PRECLINICAL DEVELOPMENT OF MEDICAL DEVICES VERSUS DRUGS, AND UNIQUE CHALLENGES WITH NCAP DEVICE DEVELOPMENT}

Small molecule compounds or protein therapeutic agents such as monoclonal antibodies developed as targeted therapies for RA and IBD are typically potent and highly selective molecules that interact with a single soluble mediator, receptor or signal transduction molecule. Characteristics of the interaction between the drug molecule and its target are traditionally first determined in in vitro assay systems. The molecule is then studied in a standard, accepted animal model, establishing a relationship between delivered dose and tissue exposure, relevant biomarker effect and measures of efficacy. This concentration-activity-effect relationship is then used to inform the choice of doses selected for human studies.

In contrast, electrically active implantable medical devices (for example, cardiac pacemakers, spinal cord stimulators for pain reduction) typically alter physiologic activity of an entire organ or organ system by inducing depolarization of excitable neural or muscle tissues in a selective manner. Depolarization is elic- 
Table 1. CAP activation and/or vagotomy in preclinical rheumatoid arthritis models.

\begin{tabular}{|c|c|c|c|c|}
\hline Intervention & Model & Species & Result & Reference \\
\hline $\begin{array}{l}\text { Cervical vagotomy; and } \\
\text { nAChR agonists nicotine } \\
\text { or AR-R17779 }\end{array}$ & $\mathrm{CIA}$ & Mouse & $\begin{array}{l}\text { Vagotomy worsens and nAChR agonists improve arthritis score. } \\
\text { nAChR agonists improve bone degradation, synovial inflammation, TNF. }\end{array}$ & $(48)$ \\
\hline$\alpha 7 n A C h R$ Knockout & $\mathrm{ClA}$ & Mouse & $\begin{array}{l}\text { Knockout worsens incidence and severity of arthritis, inflammation, joint } \\
\text { destruction and proinflammatory cytokines. Decrease in the } \\
\text { collagen-specific Th1-associated lgG2a, but not lgG1. }\end{array}$ & $(29)$ \\
\hline Surgical vagus suspension & CIA & Rat & Improves paw volume, arthritis score, bone erosions, histology, serum TNF. & $(31)$ \\
\hline Vagus nerve stimulation & $\mathrm{CIA}$ & Rat & Improves paw volume, arthritis score, histology, bone metabolic markers. & $(32)$ \\
\hline $\begin{array}{l}\text { Cervical vagotomy and } \\
\text { nicotine }\end{array}$ & $\mathrm{CIA}$ & Mouse & $\begin{array}{l}\text { Vagotomy worsens and nicotine improves arthritis score, inflammation and } \\
\text { bone destruction, TNF, IL-6 expression and HMGBI translocation. }\end{array}$ & $(27)$ \\
\hline $\begin{array}{l}\text { Cervical vagotomy and } \\
\text { nicotine }\end{array}$ & $\mathrm{CIA}$ & Mouse & $\begin{array}{l}\text { Vagotomy worsens and nicotine improves arthritis score; nicotine reduces } \\
\text { Th17cells, IL-17A and ROR } \tau \text { expression; and nicotine increases IL-4 and } \\
\text { GATA3 expression. }\end{array}$ & (28) \\
\hline
\end{tabular}

CIA, collagen-induced arthritis; HMGB1, high-mobility group protein Bl; IgG, immunoglobulin G; IL, interleukin; ROR $\gamma$, RAR-related orphan receptor $\gamma$; TNF, tumor necrosis factor; WT, wild type.

ited by applying electrical charge, which can be altered by modulating the stimulation parameters of the device including the amplitude (measured as output current), waveform, frequency and duration of the delivered electrical pulse. The time duration of each stimulation and the duty cycle (ratio between the "on" and "off" time of the device) can also be altered. Together these parameters determine the overall therapeutic effect of the device on tissue function and disease pathophysiology in appropriate animal models, which then in turn informs selection of stimulation parameters used in clinical studies.

Activating the CAP using VNS to produce a therapeutic effect in an animal model is more complex than either inducing neural depolarization alone or delivering a molecule to alter a single cellular target alone. Successful intervention using NCAP requires intermittent induction of vagus nerve depolarization, releasing neurotransmitters that modulate immune cell function and ultimately reduce proinflammatory mediator release (see Figure 1). Because the mechanism of NCAP action is unconventional, preclinical development required a nontraditional, hybrid approach between the techniques typically used in inflammation drug development and those used in neuromodulation device development. In both RA and IBD, standard wellcharacterized animal models were used, but a progression from more typical pharmacologic, genetic and anatomic manipulations to direct electrical nerve stimulation was required.

\section{NCAP EFFECT IN MODELS OF RA}

The collagen-induced arthritis (CIA) model is used in drug development to assess preclinical efficacy and help guide decisions on which candidate molecules should be advanced into clinical development for RA (25). In the classical CIA model, autoimmunity against native joint structure proteins is induced in rodents by repeated injections of bovine collagen, often in the presence of an immune-activating compound such as Freund's adjuvant. Animals rapidly develop synovitis that can be assessed either by direct visualization or caliper measurement of joint swelling. Although the correlation is imperfect as with any animal model, the pathological findings in the rodent joint during CIA exhibit a reasonable degree of similarity to those seen in RA patients, including infiltration of the synovium with immune effector cells, formation of an inflammatory synovial outgrowth termed a "pannus," damage to articular cartilage and peri-articular erosions of the bone. Improvement in these parameters induced by exposure to a test compound establishes preclinical efficacy (26).

The CIA model was utilized to provide evidence supporting the advancement of
NCAP toward clinical development (Table 1). Two studies $(27,28)$ demonstrated that surgical vagotomy worsens and the $\alpha 7 \mathrm{nAChR}$ agonist ameliorates CIA disease severity. However, four key studies definitively demonstrated the importance of the pathway in affecting the course and severity of CIA and together provided the rationale to study the therapeutic potential of NCAP in RA patients.

Owing to the critical role of the $\alpha 7 n A C h R$ in mediating the CAP effect, we studied the course of CIA in mice with targeted disruption of the $\alpha 7 \mathrm{nAChR}$ receptor gene (29). When compared with wild-type littermates, $\alpha 7 \mathrm{nAChR}$ knockout animals had a greater cumulative incidence of disease onset, accompanied by worsened clinical disease severity and radiographic evidence of bone destruction, increased histological joint inflammation, systemic monocyte chemotactic peptide (MCP)-1 and TNF levels, and increased in vitro release of Th1 cytokines from cultured splenocytes (Figures 3A, B). Consistent with the antiinflammatory effect of the CAP, clinical arthritis was exacerbated by vagotomy in CIA (30). Conversely, in another set of experiments, we demonstrated that the course and severity of CIA were ameliorated by systemic treatment with nicotine (a nonselective ligand of the $\alpha 7 \mathrm{nAChR}$ ) or with the selective $\alpha 7 \mathrm{nAChR}$ agonist AR-R17779 (Figures 3C-E) (30). 
A

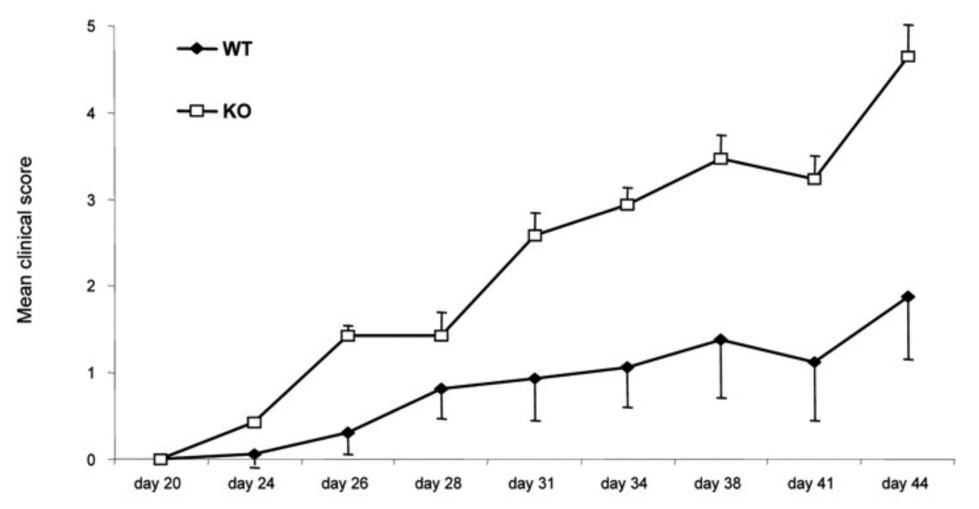

B

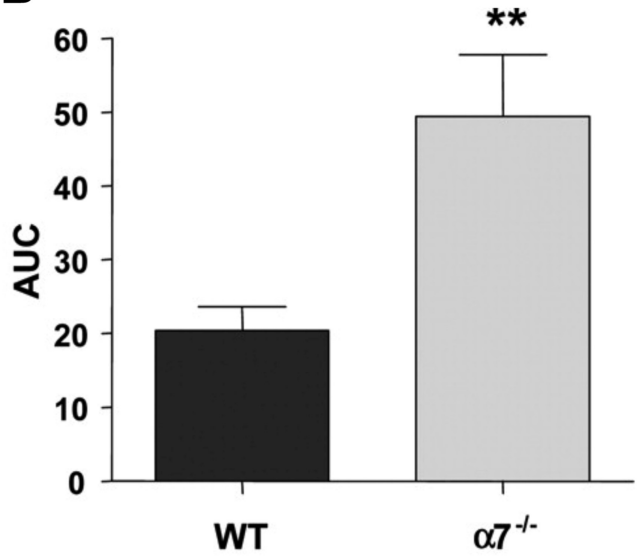

E

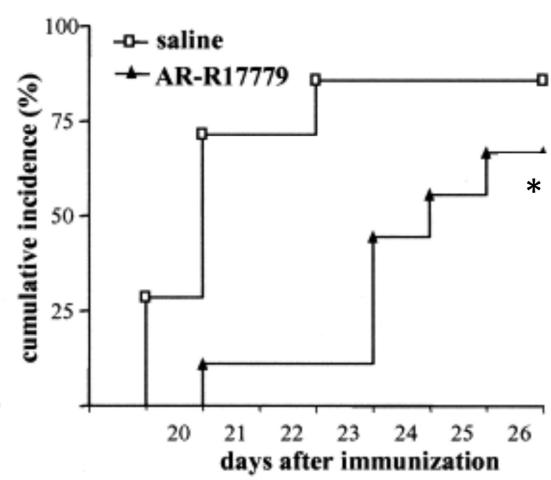

Figure 3. Both the $\alpha 7 n A C h R$ agonist AR-R17779 and nicotine improve, while targeted $\alpha 7 n A C h R$ gene disruption exacerbates CIA. CIA was induced in male and female $\alpha 7 \mathrm{nAChR}$ deficient C57BL/6 mice and wild-type littermates. Clinical arthritis scores are shown over time as mean + SE (A). Significance between groups was determined by comparing the area under the curve (AUC) from d 20 to d 26 (B), ** $P<$ 0.005 for $t$ test versus saline. Beginning on d 20 after immunization with type $\|$ collagen, male DBA/1 mice were treated with nicotine (400 g/kg; $n=8$ ) or saline, or AR-R17779 (1, 2.5, or 5 mg/kg; $n=15$ per dose group) or saline for $7 \mathrm{~d}$. Clinical arthritis scores are shown over time as mean + SE (C, D). Significance between groups was determined by comparing the AUC from d 20 to d 26 (not shown). Arthritis scores were significantly lower in mice treated with nicotine or AR-R17779 at $2.5 \mathrm{mg} / \mathrm{kg}$ or $5 \mathrm{mg} / \mathrm{kg}$ compared with scores in control animals. Incidence of arthritis in mice treated with AR-R17779 (5 mg/kg) or saline are plotted cumulatively over time $(E),{ }^{*} p \leq 0.05$ for Kaplan-Meier survival test versus saline. Panels A and B from a figure in (29) (adapted by permission from BMJ Publishing Group Limited. Annals of the Rheumatic Diseases. Role of the cholinergic nervous system in rheumatoid arthritis: aggravation of arthritis in nicotinic acetylcholine receptor $\alpha 7$ subunit gene knockout mice. Marjolein A van Maanen, Susanne P Stoof, Gregory J LaRosa, Margriet J Vervoordeldonk, Paul P Tak. 69:1717-23, ( ) 2010). Panels C-E adapted by permission from a figure in (30) (Stimulation of Nicotinic Acetylcholine Receptors Attenuates Collagen-Induced Arthritis in Mice. Marjolein A van Maanen, Maria C Lebre, Tom van der Poll, Gregory J LaRosa, Daniel Elbaum, Margriet J Vervoordeldonk, Paul P Tak. Arthritis \& Rheumatism. Vol. 60, issue 1. Copyright (c 2009 by the American College of Rheumatology).

Evidence that directly activating signaling through the vagus nerve itself could improve CIA outcome was first provided in a study using surgical suspension of the cervical portion of the nerve against the sternocleidomastoid muscle in rats (31). The apposition of muscle and nerve induced chronic mechanical vagus activation, which, when measured electrophysio- logically, appeared similar to the pattern of nerve activation achieved with direct stimulation using standard electrodes. CIA was induced in sham-operated animals and animals that underwent full surgical suspension of the nerve and both groups were followed for three months. When compared with the sham-operated group, animals with surgical suspension had statistically significant improvements in paw volume, clinical arthritis score, semiquantitative radiographic assessment of bone erosions, histological evidence of erosions and inflammation and reduced serum TNF levels.

Finally, we extended these observations, establishing the practicality and effectiveness of traditional electrical VNS in the CIA model using a chronic implantable rodent 


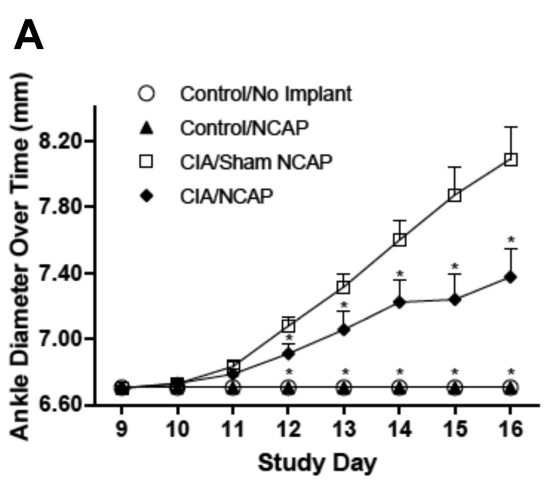

B

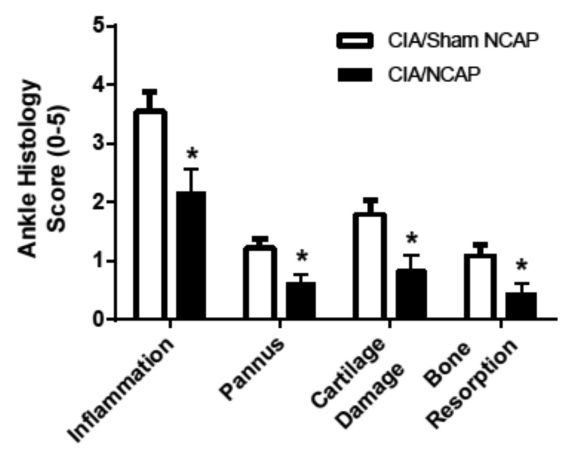

Figure 4. NCAP decreases joint swelling and histological indices of joint damage in CIA. Rats implanted with vagus nerve cuff electrodes had CIA induced or were injected with saline (Control) and were followed for $15 \mathrm{~d}$. Animals underwent active (NCAP) or sham electrical stimulation (Sham NCAP) once daily from study d 9. Ankle diameter over time is shown as mean $+\mathrm{SE}(A) .{ }^{*} P \leq 0.05$ for ANOVA versus CIA/Sham NCAP. Ankle joints were harvested on study $d 16$ and scored on a scale of 0-5 for inflammation, pannus formation, cartilage damage and bone resorption (B). Data are shown as mean + SE score. * $P \leq 0.05$ for $t$ test versus CIA/Sham NCAP. Image adapted from figures in (32) (Neurostimulation of the Cholinergic Anti-Inflammatory Pathway Ameliorates Disease in Rat Collagen-Induced Arthritis. Yaakov A Levine, Frieda A Koopman, Michael Faltys, April Caravaca, Alison Bendele, Ralph Zitnik, Margriet J Vervoordeldonk, Paul Peter Tak. PLOS ONE. 2014;9:e104530. Permission not required for adaptation. Available from: http://www.plosone.org/article/info\%3Adoi\%2F10.1371\%2Fjournal.pone.0104530).

system we developed with a cuff lead analogous to those used in humans treated with implantable VNS devices (32). In these studies, animals were immunized on d 0 and $\mathrm{d} 6$, and treatment was initiated after the disease had become semiestablished on d 9. When compared with implanted but unstimulated animals, VNS stimulation delivered at 3.0-mA output current, $10-\mathrm{Hz}$ pulse frequency, 200-microsecond pulse width for $60 \mathrm{~s}$ once daily over $\mathrm{d} 9$ through d 16 significantly reduced clinical manifestations as assessed by ankle diameter, and reduced the histological severity of inflammation, pannus formation, cartilage damage and bone resorption (Figure 4). These improvements were accompanied by a reduction in circulating proinflammatory mediators, and changes in important mediators of bone metabolism including an increase in osteoprotegerin (OPG), and a marked decrease in serum receptor activator of nuclear factor (NF)- $\kappa \mathrm{B}$ ligand

(RANKL), the major regulator of osteoclast survival, maturation, and function (33).

These observations in animal models of RA were supported by experiments in human biology models. Pretreatment of synovial tissue-derived fibroblast-like synoviocytes with acetylcholine, nicotine or AR-R17779 (all resulting in $\alpha 7 \mathrm{nAChR}$ activation) reduced production of the proinflammatory cytokines interleukin (IL)-6 and IL-8 significantly (34-36).

Moreover, it has been suggested that use of "snuff" (smokeless, nicotine-containing tobacco) is associated with lower disease activity in RA patients [37].

\section{NCAP EFFECT IN INTESTINAL INFLAMMATION MODELS}

Similar to the CIA model in RA drug development, rodent models of intestinal mucosal inflammation can be used to test preclinical efficacy and guide decisions on development of candidate molecules for use in clinical studies in inflammatory bowel disease (38). Mechanistically, the models fall broadly into four categories: (a) direct mucosal irritant-mediated inflammation (for example, dextran sulfate sodium [DSS]); (b) exposure to mucosal haptenating agents which cause delayed type hypersensitivity (DTH)-mediated in- flammation (for example, oxalazone, dinitrobenzene or trinitrobenzene sulfonic acid [DNBS/TNBS]); (c) spontaneous colon and small intestinal inflammation occurring in IL-10 knockout mice; and (d) induction of mucosal inflammation by $\mathrm{T}$ cell transfer into recombinaseactivating gene (RAG) knockout mice lacking native T cells) (39-42). Notably, none of these models by themselves fully emulate the pathology of either Crohn's disease or ulcerative colitis, and they have been generally less predictive of eventual outcomes in IBD clinical studies than the CIA model has been for RA (38). The effect of therapeutic intervention in all of these models is assessed by changes in levels of systemic inflammatory mediators, clinical assessment of weight loss, stool frequency and stool characteristics, morphometric or semiquantitative scoring of ulceration and inflammation in whole-intestine preparations, endoscopic scoring of disease, gut histology and level of inflammatory mediators and immune cell products (for example, leukocyte myeloperoxidase [MPO]) in gut tissue.

Pharmacologic activation using $\alpha 7 \mathrm{nAChR}$-selective and nonselective CAP activators, and CAP inhibition by vagotomy have been studied extensively in the mucosal irritant, hapten-mediated, and IL10 knockout models (Table 2). In general, vagotomy causes worsening in disease parameters, and pharmacologic CAP activation ameliorates disease activity. However, there are notable exceptions: Eliakim et al. (43) found that nicotine improved colitis, but worsened jejunal inflammation in the IL-10 knockout model. The authors considered this finding to be consistent with the well-accepted clinical observation that smoking tends to lessen disease activity in ulcerative colitis, and exacerbate its course in Crohn's patients. Moreover, in contrast to the CIA model findings noted above where $\alpha 7 \mathrm{nAChR}$-selective agonists have therapeutic efficacy, Snoek, et al. found that this class of agents were either ineffective, or actually exacerbated disease in a hapten model (44). Despite these conflicting observations, the preponderance of evidence from vagotomy and pharmacologic 
Table 2. CAP activation and/or vagotomy in preclinical colitis models.

\begin{tabular}{|c|c|c|c|c|}
\hline Intervention & Model & Species & Result & Reference \\
\hline Nicotine & TNBS & Rat & Improves macroscopic colon score, MPO activity, histology. & $(43)$ \\
\hline Nicotine & TNBS & Rat & $\begin{array}{l}\text { Improves clinical score, macroscopic colon score, MPO activity, histology, } \\
\text { colonic tissue LTB4 and TNF levels. }\end{array}$ & $(49)$ \\
\hline Nicotine & IL-10 knockout & Mouse & Improves clinical score and histology in colon; worsens jejunal histology. & $(50)$ \\
\hline Neostigmine & DNBS & Rat & Improves clinical score, macroscopic colon score, MPO activity, histology. & $(51)$ \\
\hline Vagotomy or nicotine & DSS & Mouse & $\begin{array}{l}\text { Vagotomy worsens and nicotine improves clinical score, macroscopic } \\
\text { colon score, MPO activity, histology, colonic tissue IL-6, SAP. }\end{array}$ & $(52)$ \\
\hline Vagotomy & DNBS & Mouse & $\begin{array}{l}\text { Vagotomy worsens clinical score, macroscopic colon score, MPO activity, } \\
\text { histology, colonic tissue IL-6 and TNF, SAP. }\end{array}$ & $(52)$ \\
\hline $\begin{array}{l}\text { nAChR agonist (AN) } \\
\text { or antagonist (CHD) }\end{array}$ & TNBS & Mouse & $\begin{array}{l}\text { nAChR antagonist worsens and nAChR agonist improves histological } \\
\text { scores, MPO, TNF and NF-kB in lamina propria mononuclear cells. }\end{array}$ & $(53)$ \\
\hline $\begin{array}{l}\text { Choline or } \alpha 7 n A c h R \\
\text { antagonist (DMI) }\end{array}$ & $\begin{array}{l}\text { DSS or DSS with } \\
\text { reactivation }\end{array}$ & Mouse & $\begin{array}{l}\text { Choline decreases macroscopic scores, MPO, CRP, IL-6 and IL-1 } \beta \text { while } \\
\alpha 7 n A c h R \text { antagonist worsens macroscopic scores and blocks } \\
\text { therapeutic benefit of antidepressant. }\end{array}$ & $(54)$ \\
\hline $\begin{array}{l}\alpha 7 \text { nAChR knockout } \\
\pm \text { vagotomy }\end{array}$ & DSS & Mouse & $\begin{array}{l}\text { Knockout and vagotomy worsen MPO, CRP, IL-6 and IL-1 } \beta \text {; and knockout } \\
\text { prevents therapeutic benefit of choline. }\end{array}$ & $(54)$ \\
\hline Vagotomy & DSS & Mouse & $\begin{array}{l}\text { Vagotomy worsens clinical and macroscopic scores, MPO activity; } \\
\text { increases colonic NF-kB signaling; increases splenic tissue levels of TNF, } \\
\text { IL-6, MCP-1; and decreases CD4 } 4^{+} \text {FoxP }{ }^{+} \text {Treg cells. Effect of vagotomy can } \\
\text { be adoptively transferred via CD4 } 4^{+} \text {CD25- T cells. }\end{array}$ & $(55)$ \\
\hline$\alpha 7-n A c h R$ agonist & DSS or TNBS & Mouse & AR-R17779 and GSK 1345038A either are ineffective or worsen disease & $(44)$ \\
\hline Nicotine & $\begin{array}{l}\text { Oxalazone or } \\
\text { TNBS }\end{array}$ & Mouse & Oxalazone colitis improves; there is no change in TNBS colitis. & $(56)$ \\
\hline
\end{tabular}

DSS, dextran sulfate sodium; MPO ,myeloperoxidase; DNBS/TNBS, dinitrobenzene/trinitrobenzene sulfonic acid; LT, leukotriene; IL, interleukin; CD, Cluster of Differentiation; Fox, forkhead box; TNF, tumor necrosis factor; MCP, monocyte chemotactic peptide; NF, nuclear factor; AN, anabaseine; CHD, chlorisondamine diiodide; DMI, desmethylimipramine; SAP, serum amyloid P.

intervention studies predicts that CAP activation may have beneficial effects on intestinal mucosal inflammation, and therefore might also be expected improve clinical outcomes in IBD.

More recent studies have used electrical VNS delivered by chronically implanted cuff leads similar to those we used in the rodent CIA models, supporting the feasibility of this approach, and providing a rationale to study NCAP delivery using implantable medical devices in IBD.

Meregnani, et al. used a smaller diameter version of an approved and commercially marketed VNS helical coil lead that was implanted on the vagus nerve in rats, and connected to a subcutaneously implanted connection block. Stimulation was delivered by external leads connected percutaneously to the implanted block in awake and mobile animals using an external pulse generator set to deliver an output current of $1-\mathrm{mA}$, at $5-\mathrm{Hz}$ pulse frequency, 500-microsecond pulse width, and a duty cycle of $10 \mathrm{~s}$ on/90 s off for $3 \mathrm{~h}$ per day (45). In these experiments, VNS was begun on the same day as initial TNBS exposure. Compared to implanted animals without VNS treatment, VNS reduced TNBS-induced reductions in body weight, colonic lesional edge MPO and histologic indices of inflammation. Lesional levels of mRNA for TNF, IL- 6 and IL-1 $\beta$ were not reduced by VNS treatment, nor were intralesional histologic inflammation scores or MPO levels.

Another investigative group reported on the effects of VNS delivered using a helical cuff electrode similarly implanted and linked to a subcutaneous connection block, which was then connected to an external pulse generator (46). TNBS colitis was induced concomitantly with VNS initiation. VNS was given for $3 \mathrm{~h}$ per day at 0.25-mA output current, 20-Hz pulse frequency, 500-microsecond pulse width, and duty cycle $30 \mathrm{~s}$ on/5 min off. VNS significantly improved TNBS-induced body weight reductions, clinical disease activity and colonic tissue histology, MPO, inducible nitric oxide synthetase, IL-6 and TNF. VNS increased acetylcholine levels in mucosal tissue, and reduced phosphorylated forms of NF-кB p65, ERK, JNK and p38 MAPK, all of which are important subcellular intermediate signaling molecules that modulate cytokine secretion (Figure 5).

\section{TAKING NCAP FROM PRECLINICAL MODELS TO HUMAN TRIALS}

As summarized above, an understanding of the neuroanatomy and immunology of the CAP and identification of key components of the signaling path (for example, the $\alpha 7 \mathrm{nAChR}$ ) enabled testing of the hypothesis that modulating the activation state of the CAP could affect disease severity in classical inflammation models of RA and IBD. In both cases, evidence of effect first came from experiments in which pharmacologic agonists or antagonists ameliorated or exacerbated disease 

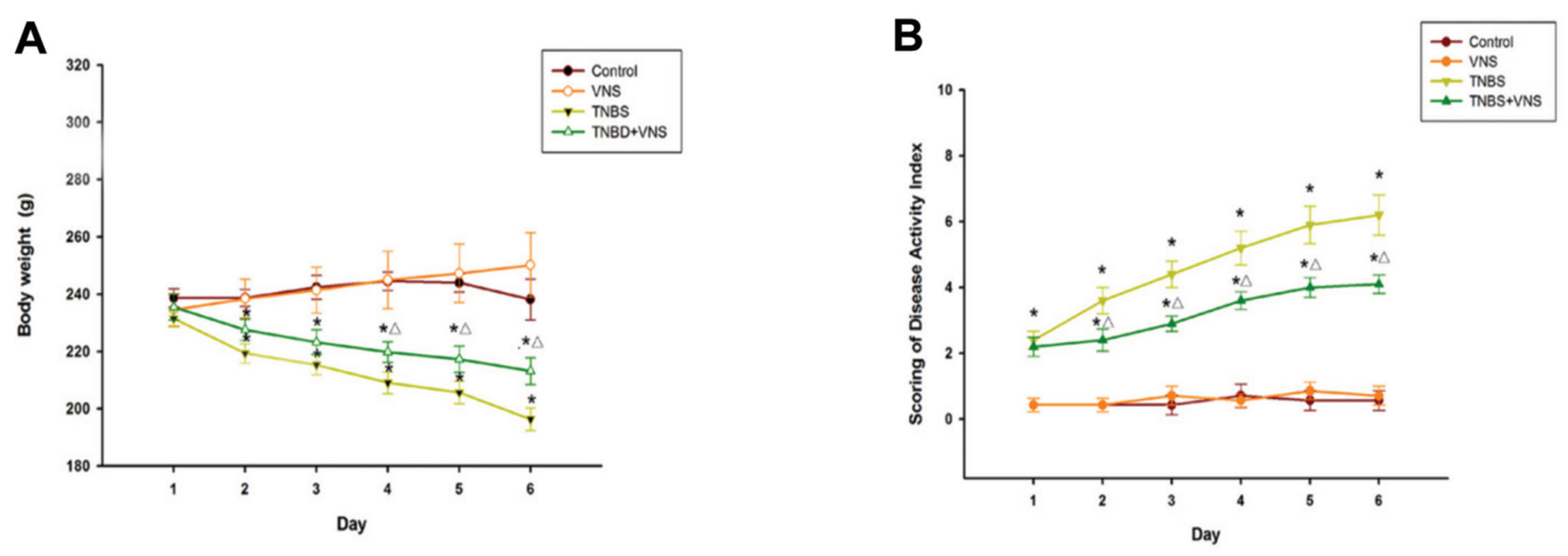

\begin{tabular}{|c|c|c|c|c|c|}
\hline C \\
\hline Group & $\mathrm{n}$ & MPO $(\mathrm{U} / \mathrm{ml})$ & iNOS $(\mathrm{U} / \mathrm{ml})$ & TNF-a $(\mathrm{pg} / \mathrm{ml})$ & IL-6 $(\mathrm{pg} / \mathrm{ml})$ \\
\hline Control & 8 & $1.76 \pm 0.16$ & $1.27 \pm 0.75$ & $98.31 \pm 14.87$ & $105.47 \pm 13.56$ \\
\hline VNS & 8 & $1.89 \pm 0.18$ & $0.93 \pm 0.23$ & $101.53 \pm 11.95$ & $106.64 \pm 11.47$ \\
\hline TNBS & 10 & $2.90 \pm 0.36^{*}$ & $2.20 \pm 1.05^{*}$ & $195.21 \pm 10.76^{*}$ & $183.89 \pm 9.77^{*}$ \\
\hline TNBS+VNS & 10 & $2.09 \pm 0.33^{* \#}$ & $1.18 \pm 0.58^{*}$ & $151.21 \pm 8.53^{* \#}$ & $137.96 \pm 8.04^{* \#}$ \\
\hline
\end{tabular}

Figure 5. NCAP reduces weight loss, disease activity level, and systemic and intestinal inflammatory mediator levels in TNBS colitis. Rats implanted with vagus nerve cuff electrodes had colitis induced by intrarectal administration of TNBS or received saline control, and were followed for $6 \mathrm{~d}$. Rats then received VNS or sham VNS for $3 \mathrm{~h}$ per day from experimental d 1 to d $6(0.25 \mathrm{~mA}, 20 \mathrm{~Hz}, 500-\mathrm{ms}$ pulse width, $30 \mathrm{~s}$ on, 5 min off continuously). Body weight (A) and disease activity index (DAI) (B) are shown as mean \pm SE. MPO, inducible nitric oxide synthase (iNOS), TNF and IL-6 were measured from d-6 colonic homogenates (C). ${ }^{*} P<0.05$ versus the control group and VNS group; ${ }^{\Delta} P<0.05$ versus the TNBS group (B). MPO (U/mL), iNOS activity $(\mathrm{U} / \mathrm{mL})$, TNF- $\alpha$ (pg/mg tissue) and IL-6 levels after $6 \mathrm{~d}$ of VNS in TNBS rats. Data presented as mean \pm SE. ${ }^{*} P<0.05$ versus control group; ${ }^{\#} P<0.05$ versus TNBS group (C). Image adapted from a figure and a table in (46) (Involvement of MAPK/NF-KB Signaling in the Activation of the Cholinergic Anti-Inflammatory Pathway in Experimental Colitis by Chronic Vagus Nerve Stimulation. Peng Sun, Kewen Zhou, Sheng Wang, Ping Li, Sijuan Chen, Guiping Lin, Yan Zhao, Tinghuai Wang. PLOS ONE. 2013:8:e69424. Permission not required for adaptation. Available from: http://www.plosone.org/article/info\%3Adoi\%2F10.1371\%2Fjournal.pone.0069424).

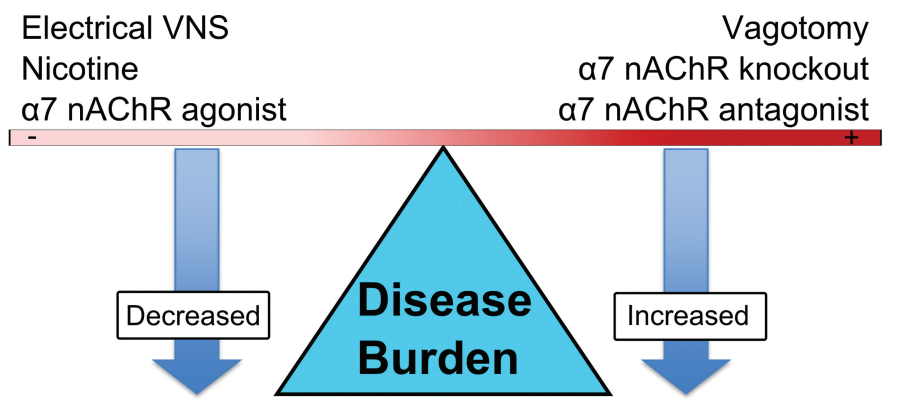

Figure 6. Experimental manipulation of the cholinergic inflammatory pathway influences disease burden in preclinical models of RA and IBD. Surgical, genetic, or pharmacologic disruption of the CAP increases, while electrical and pharmacologic stimulation of the CAP decreases disease burden in preclinical models of RA and IBD. manifestations, and from demonstrating the exacerbating effect of blocking the pathway by anatomic destruction of the involved nerves or targeted disruption of the $\alpha 7 \mathrm{nAChR}$ gene. Finally, progression from pharmacologic, anatomic, or genetic experiments to studies of the effect of direct stimulation of the vagus nerve provided further confirmation. The summation of the evidence for the importance of the pathway in improving or exacerbating disease burden these models is shown schematically in Figure 6. Indeed on the basis of this body of evidence there was a 
clear rationale to study electrically active implantable vagus nerve stimulation devices in RA and IBD. Early studies by our group and others are underway (NCT 01569503, NCT 01552941), and further definitive studies are being planned.

\section{TRANSLATION OF BIOELECTRONIC MEDICINES-OPPORTUNITIES AND CHALLENGES}

An effort to increase the therapeutic armamentarium in systemic diseases typically treated with systemically administered drugs has resulted in the concept of bioelectronic medicines (47). Such therapies would have structural and functional characteristics of both classical medicines and classical medical devices. They would be embedded within the body, and would deliver electrical or other kinds of physical stimulation in a precise and targeted manner that would directly affect the disease pathophysiology. Ideally the therapeutic entity would be able to monitor the function of the tissue or organ system continuously, adjusting its therapeutic effect in a precise way that would maximize safety and efficacy.

To produce a therapy that fulfills all of these requirements, significant further advances in basic science and engineering will be necessary. However, the use of NCAP for RA and IBD represents an early step in the search for an ideal bioelectronic medicine, and challenges faced during development of NCAP can be of help in guiding future efforts. For example, our experience indicates that great attention must be paid to creating implanted hardware that correctly fits the anatomy of and interacts with the model species in a reliable and robust way, and to ensuring the effects of the surgery and the chronic implant do not interfere with biomarkers and disease measurements. Further, in contrast to drug development where the relationship between pharmacokinetic and pharmacodynamic parameters and disease activity in the model is often well understood, with implanted devices this is usually not the case. Similarly, the optimal way to extrapolate device stimulation parameters from animal to human studies in complex systemic diseases is not yet well studied.

Despite these challenges, the prospect of creating an entirely new way to treat serious systemic illnesses with high medical need such as RA and IBD remains exciting. Along the way to development of an idealized bioelectronic medicine, NCAP therapy is now being studied to determine if it can fulfill its promise of providing an alternative to drug therapy, with a potential for lower cost and better safety, thus better fulfilling the needs of patients, providers and health-care payers.

\section{DISCLOSURE}

YA Levine, M Faltys and R Zitnik are employees of SetPoint Medical Corporation. P-P Tak is a consultant to and has received research grants from SetPoint Medical and is an employee of GlaxoSmithKline.

\section{REFERENCES}

1. Scott DL, Wolfe F, Huizinga TW. (2010) Rheumatoid arthritis. Lancet. 376:1094-108.

2. Cheifetz AS. (2013) Management of active Crohn disease. JAMA. 309:2150-8.

3. Gkaliagkousi E, et al. (2012) Cardiovascular risk in rheumatoid arthritis: pathogenesis, diagnosis, and management. J. Clin. Rheumatol. 18:422-30.

4. Andersson U, Tracey KJ. (2012) Reflex principles of immunological homeostasis. Annu. Rev. Immunol. 30:313-35.

5. Tracey KJ. (2009) Reflex control of immunity. Nat. Rev. Immunol. 9:418-428.

6. Watkins LR, et al. (1995) Blockade of interleukin-1 induced hyperthermia by subdiaphragmatic vagotomy: evidence for vagal mediation of immunebrain communication. Neurosci. Lett. 183:27-31.

7. Bianchi M, et al. (1995) An inhibitor of macrophage arginine transport and nitric oxide production (CNI-1493) prevents acute inflammation and endotoxin lethality. Mol. Med. 1:254-266.

8. Borovikova L, et al. (2000) Vagus nerve stimulation attenuates the systemic inflammatory response to endotoxin. Nature. 405:458-62.

9. Huston JM, et al. (2006) Splenectomy inactivates the cholinergic antiinflammatory pathway during lethal endotoxemia and polymicrobial sepsis. J. Exp. Med. 203:1623-8.

10 Rosas-Ballina M, et al. (2008) Splenic nerve is required for cholinergic antiinflammatory pathway control of TNF in endotoxemia. Proc. Natl. Acad. Sci. U. S. A. 105:11008-13.

11. Rosas-Ballina M, et al. (2011) Acetylcholinesynthesizing $\mathrm{T}$ cells relay neural signals in a vagus nerve circuit. Science. 334:98-101.
12. Wang H, et al. (2003) Nicotinic acetylcholine receptor [alpha]7 subunit is an essential regulator of inflammation. Nature. 421:384-8.

13. Olofsson PS, et al. (2012) alpha7 nicotinic acetylcholine receptor (alpha7nAChR) expression in bone marrow-derived non-T cells is required for the inflammatory reflex. Mol. Med. 18:539-43.

14. Matteoli G, Boeckxstaens GE. (2013) The vagal innervation of the gut and immune homeostasis. Gut. 62:1214-22.

15. Matteoli G, et al. (2014) A distinct vagal antiinflammatory pathway modulates intestinal muscularis resident macrophages independent of the spleen. Gut. 63:938-4.

16. Ji H, et al. (2014) Central cholinergic activation of a vagus nerve-to-spleen circuit alleviates experimental colitis. Mucosal Immunol. 7:335-47

17. Bruchfeld A, et al. (2010) Whole blood cytokine attenuation by cholinergic agonists ex vivo and relationship to vagus nerve activity in rheumatoid arthritis. J. Intern. Med. 268:94-101.

18. Parrish WR, et al. (2008) Modulation of TNF release by choline requires alpha7 subunit nicotinic acetylcholine receptor-mediated signaling. Mol. Med. 14:567-74.

19. de Jonge WJ, et al. (2005) Stimulation of the vagus nerve attenuates macrophage activation by activating the Jak2-STAT3 signaling pathway. Nat. Immunol. 6:844-51.

20. van Westerloo DJ, et al. (2006) The vagus nerve and nicotinic receptors modulate experimental pancreatitis severity in mice. Gastroenterology. 130:1822-30.

21. Krzyzaniak MJ, et al. (2011) Efferent vagal nerve stimulation attenuates acute lung injury following burn: The importance of the gut-lung axis. Surgery. 150:379-89

22. Huston JM, et al. (2009) Cholinergic neural signals to the spleen down-regulate leukocyte trafficking via CD11b. J. Immunol. 183:552-9.

23. Van Maanen M, Vervoordeldonk M, Tak P. (2009) The cholinergic anti-inflammatory pathway: towards innovative treatment of rheumatoid arthritis. Nat. Rev. Rheumatol. 5:229-32.

24. Koopman FA, et al. (2011) Restoring the balance of the autonomic nervous system as an innovative approach to the treatment of rheumatoid arthritis. Mol. Med. 17:937-48.

25. Bevaart L, Vervoordeldonk MJ, Tak PP. (2010) Evaluation of therapeutic targets in animal models of arthritis: how does it relate to rheumatoid arthritis? Arthritis Rheum. 62:2192-205.

26. Hegen M, Keith JC Jr, Collins M, NickersonNutter CL. (2008) Utility of animal models for identification of potential therapeutics for rheumatoid arthritis. Ann. Rheum. Dis. 67:1505-15.

27. Li T, et al. (2010) The vagus nerve and nicotinic receptors involve inhibition of HMGB1 release and early pro-inflammatory cytokines function in collagen-induced arthritis. J. Clin. Immunol. 30:213-20.

28. Wu S, et al. (2014) Attenuation of collagen induced arthritis via suppression on Th17 response 
by activating cholinergic anti-inflammatory pathway with nicotine. Eur. J. Pharmacol. 735:97-104.

29. van Maanen M, Stoof S, Larosa G, Vervoordeldonk M, Tak P. (2010) Role of the cholinergic nervous system in rheumatoid arthritis: aggravation of arthritis in nicotinic acetylcholine receptor alpha7 subunit gene knockout mice. Ann. Rheum. Dis. 69:1717-23.

30. van Maanen MA. (2009) Stimulation of nicotinic acetylcholine receptors attenuates collagen-induced arthritis in mice. Arthritis Rheum. 60:114-122.

31. Zhang P, Han D, Tang T, Zhang X, Dai K. (2008) Inhibition of the development of collagen-induced arthritis in Wistar rats through vagus nerve suspension: a 3-month observation. Inflamm. Res. 57:322-328.

32. Levine YA, et al. (2014) Neurostimulation of the cholinergic anti-inflammatory pathway ameliorates disease in rat collagen-induced arthritis. PLoS One. 9:e104530.

33. Lacey DL, et al. (2012) Bench to bedside: elucidation of the OPG-RANK-RANKL pathway and the development of denosumab. Nat. Rev. Drug. Discov. 11:401-19.

34. Zhou Y, et al. (2012) Nicotine inhibits tumor necrosis factor-alpha induced IL- 6 and IL-8 secretion in fibroblast-like synoviocytes from patients with rheumatoid arthritis. Rheumatol. Int. 32:97-104.

35. van Maanen MA, et al. (2009) The alpha7 nicotinic acetylcholine receptor on fibroblast-like synoviocytes and in synovial tissue from rheumatoid arthritis patients: a possible role for a key neurotransmitter in synovial inflammation. Arthritis Rheum. 60:1272-81.

36. Waldburger JM, Boyle DL, Pavlov VA, Tracey KJ, Firestein GS. (2008) Acetylcholine regulation of synoviocyte cytokine expression by the alpha7 nicotinic receptor. Arthritis Rheum. 58:3439-49.

37. Andersson ML, Bergman S, Soderlin MK, BARFOT study group (2013) The effect of snuff (smokeless tobacco) on disease activity and function in rheumatoid arthritis: experiences from the Better Anti-Rheumatic FarmacOTherapy, a longitudinal multicenter study on early rheumatoid arthritis. J. Clin. Rheumatol. 19:14-18.

38. Valatas V, Vakas M, Kolios G. (2013) The value of experimental models of colitis in predicting efficacy of biological therapies for inflammatory bowel diseases. Am. J. Physiol. Gastrointest. Liver Physiol. 305:G763-85.

39. Ostanin DV, et al. (2009) T cell transfer model of chronic colitis: concepts, considerations, and tricks of the trade. Am. J. Physiol. Gastrointest. Liver Physiol. 296:G135-46.

40. Perse M, Cerar A. (2012) Dextran sodium sulphate colitis mouse model: traps and tricks. J. Biomed. Biotechnol. 2012:718617.

41. te Velde AA, Verstege MI, Hommes DW. (2006) Critical appraisal of the current practice in murine TNBS-induced colitis. Inflamm Bowel Dis 12:995-9.

42. Rennick DM, Fort MM. (2000) Lessons from genetically engineered animal models. XII. IL-10deficient (IL-10(-/-) mice and intestinal inflam- mation. Am. J. Physiol. Gastrointest. Liver Physiol. 278:G829-33.

43. Eliakim R, Karmeli F, Rachmilewitz D, Cohen P, Fich A. (1998) Effect of chronic nicotine administration on trinitrobenzene sulphonic acid-induced colitis. Eur. J. Gastroenterol. Hepatol. 10:1013-9.

44. Snoek SA, et al. (2010) Selective alpha7 nicotinic acetylcholine receptor agonists worsen disease in experimental colitis. Br. J. Pharmacol. 160:322-33.

45. Meregnani J, et al. (2011) Anti-inflammatory effect of vagus nerve stimulation in a rat model of inflammatory bowel disease. Auton. Neurosci. 160:82-9.

46. Sun P, et al. (2013) Involvement of MAPK/NF-кB signaling in the activation of the cholinergic antiinflammatory pathway in experimental colitis by chronic vagus nerve stimulation. PLoS One. 8:e69424.

47. Famm K, Litt B, Tracey KJ, Boyden ES, Slaoui M. (2013) Drug discovery: a jump-start for electroceuticals. Nature. 496:159-61.

48. van Maanen M, et al. (2009) Stimulation of nicotinic acetylcholine receptors attenuates collagen-induced arthritis in mice. Arthritis Rheum. 60:114-22.

49. Sykes AP, et al. (2000) An investigation into the effect and mechanisms of action of nicotine in inflammatory bowel disease. Inflamm. Res. 49:311-9.

50. Eliakim R, Fan QX, Babyatsky MW. (2002) Chronic nicotine administration differentially alters jejunal and colonic inflammation in interleukin-10 deficient mice. Eur. J. Gastroenterol. Hepatol. 14:607-14.

51. Miceli PC, Jacobson K. (2003) Cholinergic pathways modulate experimental dinitrobenzene sulfonic acid colitis in rats. Auton. Neurosci. 105:16-24.

52. Ghia JE, Blennerhassett $P$, Kumar-Ondiveeran $H$, Verdu EF, Collins SM. (2006) The vagus nerve: a tonic inhibitory influence associated with inflammatory bowel disease in a murine model. Gastroenterology. 131:1122-30

53. Bai A, Guo Y, Lu N. (2007) The effect of the cholinergic anti-inflammatory pathway on experimental colitis. Scand. J. Immunol. 66:538-45.

54. Ghia JE, et al. (2009) Reactivation of inflammatory bowel disease in a mouse model of depression. Gastroenterology. 136:2280-8.

55. O'Mahony C, van der Kleij H, Bienenstock J, Shanahan F, O'Mahony L. (2009) Loss of vagal anti-inflammatory effect: in vivo visualization and adoptive transfer. Am. J. Physiol. Regul. Integr. Comp. Physiol. 297:R1118-26.

56. Galitovskiy V, et al. (2011) Cytokine-induced alterations of alpha7 nicotinic receptor in colonic $\mathrm{CD} 4 \mathrm{~T}$ cells mediate dichotomous response to nicotine in murine models of Th1/Th17- versus Th2-mediated colitis. J. Immunol. 187:2677-87.

Cite this article as: Levine YA, Koopman F, Faltys M, Zitnik R, Tak P-P. (2014) Neurostimulation of the cholinergic antiinflammatory pathway in rheumatoid arthritis and inflammatory bowel disease. Bioelectron. Med. 1:34-43. 